Smith, M. H., \& Duffy, M. Consumption of sucrose and saccharin by hungry and satiated rats. Journal of Comparative and Physiological Psychology, 1957, 50, 65-69.

Ternes, $J$. W. induced preference for morphine in rats. Bulletin of the Psychonomic Society, 1975a, in press.

Ternes, J. W. Naloxone induced aversion to sucrose in morphine dependent rats. Bulletin of the Psychonomic Society, 1975b, in press.

Weisinger, R. S., Parker, L. F., \& Skorupski, J. D. Conditioned taste aversions and specific needs states in the rat. Journal of
Comparative and Physiological Psychology, 1974, 87, 655-660.

Winer, B. J. Statistical principles in experimental design. New York: McGraw-Hill, 1962.

Wolf, G. Innate mechanisms for regulation of sodium intake. In C. Pfaffmann (Ed.), Olfaction and taste. New York: Rockefeller University Press, 1969.

Bulletin of the Psychonomic Society

1975, Vol. 5 (4), 294-296

\title{
Alley section effects of magnitude of partial reward after extensive acquisition training
}

\author{
E. J. CAPALDI and MICHAEL R. FREESE \\ Purdue University, West Lafayette, Indiana 47907
}

\begin{abstract}
Rats were given either a small or a large magnitude of partial reward (100 trials) in a runway and 50 extinction trials. The initial trial acquisition superiority associated with the larger magnitude of partial reward maintained itself throughout acquisitions in the start section of the runway but disappeared by the last block of 25 trials in the run section and following the first block of 25 trials in the goal section. In extinction there was a marginal tendency, totally absent in the start and goal sections, for resistance to extinction to increase as magnitude of partial reward increased. These results suggest that the early trial acquisition and extinction superiority associated with larger magnitudes of partial reward are reduced by late trial inhibitory effects which increase toward the goal section and as magnitude of partial reward increases.
\end{abstract}

The effects of magnitude of partial reward on acquisition and extinction appear to depend upon level of acquisition training (see Capaldi \& Freese, 1974). That is, both the acquisition and extinction superiority associated with larger magnitudes of partial reward earlier in acquisition training tend to disappear following more extensive training (McCain, 1970), and, indeed, following extensive acquisition training, resistance to extinction may actually decrease as magnitude of partial reward increases (Campbell, Crumbaugh, Rhodus, \& Knouse, 1971; Capldi \& Freese, 1974).

There is reason to suppose (see Capaldi \& Levy, 1972) that the effects of magnitude of partial reward on acquisition and extinction following extensive acquisition training may depend upon section of the runway but data on this point are not available. McCain (1970) did not report alley section findings and the section findings of Campbell et al. (1971), while suggestive, may be peculiar to the alternating schedule of reward and nonreward employed in that investigation. This investigation employed a large vs. a small magnitude of partial reward on an irregular training schedule for 100 acquisition trials, followed by 50 extinction trials with performance being measured over the early (start),

This research was supported in part by National Institute of Child Health Development Grant 5R HD 04379-03 to the first author and by a Purdue University David Ross predoctoral Fellowship to the second author.

Requests for reprints should be sent to E. J. Capaldi, Department of Psychological Sciences, Purdue University, West Lafay ette, Indiana 47907. middle (run) and late (goal) portions of the alley.

\section{METHOD}

\section{Subjects}

The subjects were 20 male albino rats, 100 days old at the beginning of the experiment, purchased from the Holtzman Co., Madison, Wisconsin. The mean weights of the animals were monitored and the two groups did not differ in weight either at the beginning of acquisition $[F(1,18)=1.88, p>.20]$ or on the first day of extinction $(F<1)$.

\section{Apparatus}

The gray runway was $208.4 \mathrm{~cm}$ long, $10.2 \mathrm{~cm}$ wide, with $22.9 \mathrm{~cm}$ high sides. It was covered with $1.3-\mathrm{cm}$ hardware cloth. A $20.3-\mathrm{cm}$ long floor treadle suspended over the microswitch constituted the initial portion of the alley. When the treadle was depressed by the rat, whose front paws were always placed at the extreme forward edge, the first clock $(.01 \mathrm{sec})$ was started. Interrupting a photobeam $5.1 \mathrm{~cm}$ beyond the treadle stopped Clock 1 (start time) and started Clock 2. Interrupting a photobeam $131.1 \mathrm{~cm}$ beyond the first photobeam stopped Clock 2 (running time) and started Clock 3. Interrupting a photobeam $30.4 \mathrm{~cm}$ beyond the second photobeam and $6.4 \mathrm{~cm}$ in front of the brass $5.1 \times 10.2 \times 3.8 \mathrm{~cm}$ foodcup stopped Clock 3 (goal time). The brass cup contained a metal inset, $3.8 \times 3.2 \times 1.9 \mathrm{~cm}$, which contained the reward pellets on goalbox placements in pretraining and on all reward trials during acquisition training. A guillotine-type brass door prevented retracing back into the runway once the rat interrupted the third photobeam in the goal section of the alley.

\section{Procedure \\ Upon arrival at the laboratory, subjects were housed in individual cages where the food and water was supplied on an} ad-lib basis for 5 days. Deprivation, which consisted of $13 \mathrm{~g} /$ day 


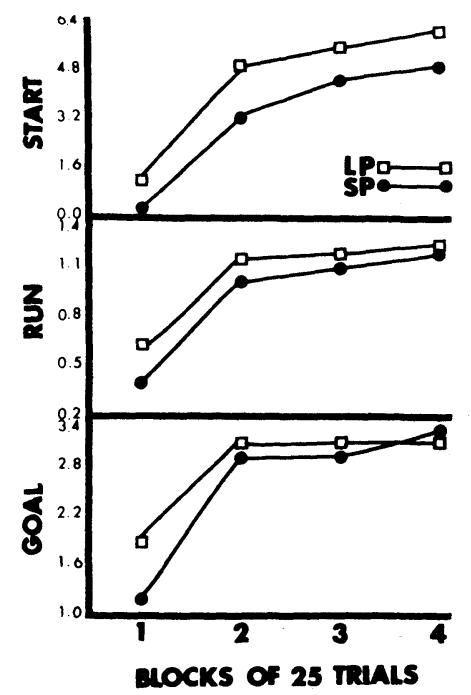

Figure 1. Mean acquisition speeds for Group LP and Group SP, in blocks of 25 trials, in the start, run, and goal sections of the alley.

of Wayne Lab Blox began on Day 1 of pretraining. Water continued to be supplied on an ad-lib basis in the home cage and the holding cages. The subjects were handled individually briefly each day from the beginning of the deprivation period. On Days $10-12$, subjects received either 2 (Groups SP) or 20 (Group LP) .045 -g Noyes pellets in the home cage. The number of pellets eaten either in the home cage or in the apparatus was always subtracted from the $13 \mathrm{~g} /$ day ration. On Day 13, each subject was given two reward placements in the goalbox, either 2 pellets reward (Group SP) or 20 pellets reward (Group LP).

Animals received five partially rewarded trials a day for 20 days. The 4-day schedule of reward $(R)$ and nonreward $(N)$, repeated five times, was RNRNR, RNNRR, RRNNR, and RNNNR. Subjects in Group SP received 2 and those in Group LP $20.045-\mathrm{g}$ Noyes pellets on each reward trial, and all subjects were removed from the goalbox once eating was finished (approximately $10 \mathrm{sec}$ for Group SP and $50 \mathrm{sec}$ for Group LP). On $\mathrm{N}$ trials, subjects were confined in the goalbox for $20 \mathrm{sec}$. During the intertrail interval (ITI) subjects were removed to metal holding cages. Following acquisition training, all animals received five nonrewarded trials a day for 10 days in extinction. Running order within and between squads was varied each day and squad composition (two subjects from each group) remained constant. The ITI was maintained at 3-7 min by giving all subjects in a squad trial $\mathrm{n}$ before any subject in that squad received trial $n+1$.

\section{RESULTS}

\section{Acquisition}

All times in each alley section were converted to speeds $(1 / \mathrm{sec})$. Mean running speeds for the start, run, and goal sections for Group LP and SP are presented in blocks of 25 trials in Figure 1. A trials by days by blocks of 25 trials by reward magnitude analysis of variance revealed that a direct relationship between reward magnitude and running speeds persisted throughout acquisition in the alley's start section, as is indicated by a significant reward magnitude effect $[F(1,118)=12.73$, $\mathrm{p}<.005$ ] and a nonsignificant Reward by Magnitude by Blocks interaction $[F(3,54)=1.60, p>.10]$. The effect of reward magnitude was not as persistent, however, in the alley's run section as in the start section. While Group LP ran significantly faster than Group SP when all acquisition training is considered $[\mathrm{F}(1,18)=5.22$, $\mathrm{p}<.05]$, the near-significant Reward Magnitude by Blocks of 25 Trials interaction $[\mathrm{F}(3,54)=2.62, \mathrm{p}=$ .059] indicated that the difference between Groups LP and SP decreased with increased acquisition training. A breakdown of the Reward Magnitude by Blocks interaction, evaluating the simple effects of reward magnitude at each 25 trial block, indicated that Group LP ran faster than Group SP over the first three 25 trials blocks $[\mathrm{Fs}(1,29)=9.18,4.85$, and 3.75 , ps $<.01, .05$, and .05 , respectively] but also that there was no difference between the groups in the final 25 trial block $(F<1)$. The effect of increased reward magnitude was even less persistent in goal lasting only over the first 25 trial block. In the goal section reward magnitude was not significant $(F<1)$ but the Reward Magnitude by Blocks interaction was significant $[F(3,54)=5.41, p<.005]$. An assessment of the effects of reward magnitude at each 25 trial block in the goal section indicated that while Group LP ran faster than Group SP in the first 25 trial block $[F(1,33)=7.33$, $p<.05]$, the two groups did not differ thereafter (Fs $<1)$.

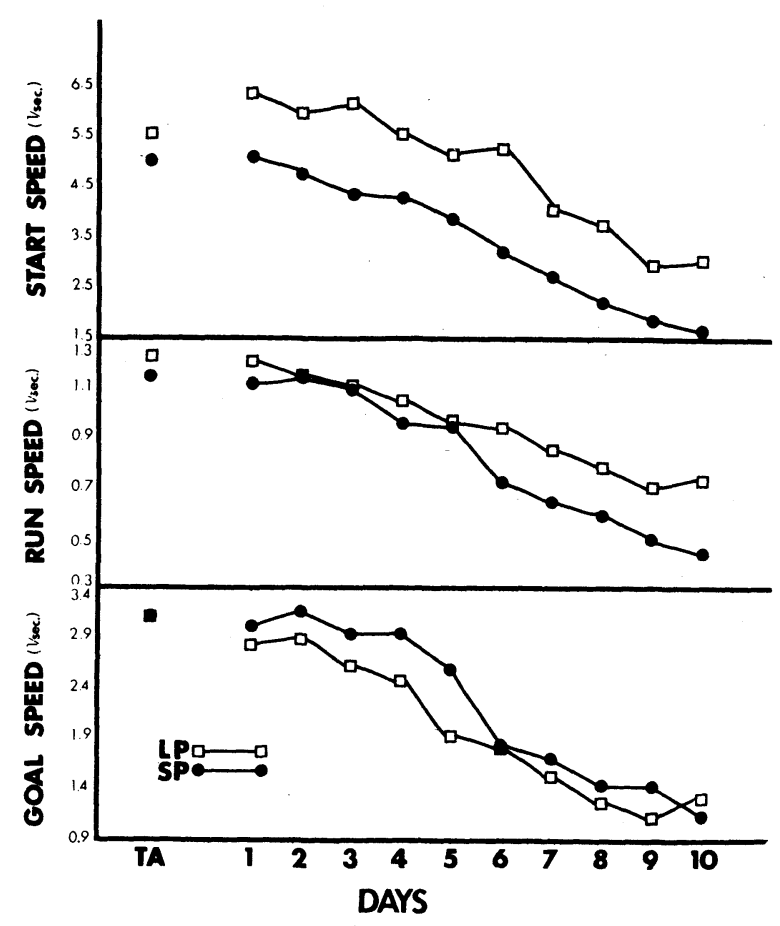

Figure 2. Mean daily extinction speeds in the start, run and goal sections of the alley for Group LP and Group SP. 


\section{Extinction}

Figure 2 shows mean daily running speeds for Groups LP and SP in the alley's start, run and goal sections. Although a Reward Magnitude by Trial by Days analysis of variance indicated that Group LP ran faster than Group SP throughout extinction in the start section of the alley $[F(1,18)=13.54, p<.005]$, the nonsignificant Reward Magnitude by Days interaction $(F<1)$, coupled with the significant advantage of Group LP over Group SP at the end of acquisition, suggests that the extinction difference merely reflects a continuation of the terminal acquisition differences in that alley section. Reward magnitude, then, did not differentially affect the rate of extinction in the start section. Similarly, in the goal section, reward magnitude did not affect rate of extinction, as neither the reward magnitude main effect $(\mathrm{F}<1)$ nor the Reward Magnitude by Days interaction $[F(9,162)=1.03, p>.20]$ were significant.

While the reward magnitude main effect was not significant in the alley-s run section $[\mathrm{F}(1,18)=2.29$, $\mathrm{p}>.10]$, the near significant Reward Magnitude by Days interaction $[\mathrm{F}(9,162)=1.90, \mathrm{p}=.055]$, combined with the lack of significant terminal acquisition differences between Group LP and SP in the run section suggests that in the run section, the running speeds of Groups LP and SP diverged slightly over extinction days. An analysis of the simple effects of reward magnitude at each day of extinction indicates that Group LP ran significantly faster than Group SP only on Day 10 of extinction $[F(1,18)=5.68, p<.05]$. No other daily differences were significant. Thus increased reward magnitude led to a marginally decreased rate of extinction in the alley's run section but did not differentially affect performance in start or goal.

\section{DISCUSSION}

Early in acquisition training in this investigation, which employed an irregular schedule of partial reward, running was faster the larger the magnitude of partial reward in all alley sections. This tendency persisted throughout acquisition training in the start section of the alley. However, it disappeared following the first block of 25 trials in the goal section of the alley and by the last block of 25 trials in the run section. The tendency for running speeds to become independent of magnitude of partial reward was thus quite orderly in this investigation, disppearing first in the goal section, later in the run section, and not at all in the start section, Perhaps no amount of training will eliminate speed differences as a function of magnitude of partial reward in the start section.

Running speeds in extinction were independent of magnitude of partial reward in the start and goal sections of the alley, and in the run section of the alley there was small and relatively minor tendency for resistance to extinction to increase over trials as the magnitude of partial reward increased. The reward magnitude main effect was not significant in the run section. Following fewer than the 100 acquisition trials employed here resistance to extinction has 'usually been found to be greater the larger the magnitude of partial reward (e.g., Capaldi \& Capaldi, 1970; Hulse, 1958; Leonard, 1969; Wagner, 1961).

According to the sequential view (e.g., Capaldi, 1966, but see especially Capaldi, 1974), stimuli are conditioned more strongly to the instrumental reaction the larger the magnitude of reward. This assumption explains why early in acquisition training running is faster the larger the magnitude of partial reward and why following limited to moderate acquisition training resistance to extinction increases as the magnitude of partial reward increases (see e.g., Capaldi, 1967). According to the sequential view, later in training conditioned inhibition is generated on nonrewarded trials. This inhibition reduces response strength and is greater the larger the magnitude of reward on the rewarded trials of the partial reward schedule (see Capaldi, 1967; Capaldi $\&$ Freese, 1974). Thus, late in training while there is more associative strength the larger the magnitude of partial reward, there is also more inhibition the larger the magnitude of partial reward; this explains presumably why running speeds become independent of magnitude of partial reward in both acquisition and extinction (see Capaldi \& Freese, 1974). Inhibition is generated in the presence of the goal cues, and would be expected, as found here, to have its initial and greatest effects in the goal section of the alley. According to Miller (1948, see also Capaldi \& Levy, 1971), the gradient of generalization for inhibition is steep. On the assumption that inhibitory effects are weak in the start section of the alley, it can be understood why running speeds in acquisition were greater the larger the magnitude of partial reward in that section following extensive training。

\section{REFERENCES}

Campbell, P. E., Crumbaugh, C. M., Rhodus, D. M., \& Knouse, S. B. Magnitude of partial reward and amount of training in the rat: A hypothesis of sequential effects. Journal of Comparative and Physiological Psychology, 1971, 75, $120-128$.

Capaldi, E. J. Partial reinforcement: A hypothesis of sequential effects. Psychological Review, 1966, 73, 459-477.

Capaldi, E. J. A sequential hy pothesis of instrumental learning. In K. W. Spence \& J. T. Spence (Eds.), The psychology of learning and motivation: Advances in research and theory. Vol. 1., New York: Academic Press, 1967, 67-156.

Capaldi, E. J. Partial reward either following or preceding consistent reward: A case of reinforcement level. Journal of Experimental Psychology, 1974, 102, 954-962.

Capaldi, E. J., \& Capaldi, E. D. Magnitude of partial reward irregular reward schedules, and a 24hour ITI: A test of several hypotheses. Journal of Comparative and Physiological Psychology, 1970 72, 203-209.

Capaldi, E. J., \& Freese, M. R. Partial reward training level and reward magnitude: Effects on acquisition and extinction. Learning and Motivation, 1974, 5, 299-310.

Capaldi, E. J., \& Levy, W. J. Stimulus control of punished reactions: Sequence of punishment trials and magnitude of reinforced trials. Learning and Motivation, 1972, 3, 1-19.

Hulse, S. H. Amount and percentage of reinforcement and duration of goal confinement in conditioning and extinction. Journal of Experimental Psychology, 1958, 56, 48-57.

Leonard, D. W. Amount and sequence of reward in partial and continuous reinforcement. Journal of Experimental Psychology, 1958, 56, 48-57.

McCain, G. Reward magnitude and instrumental responses: Consistent and partial reward. Psychonomic Science, 1970, 19, 139-141.

Miller, N. E. Theory and experiment relating psychoanalytic displacement to stimulus-response generalization. Journal of Abnormal and Social Psychology, 1948, 43, 155-177.

Wagner, A. R. Effects of amount and percentage of reinforcement and number of acquisition trials on conditioning and extinction. Journal of Experimental Psychology, 1961, 63, 234-242.

(Received for publication December 5, 1974.) 